\title{
Topographic Control of Order in Quasi-2D Granular Phase Transitions
}

\author{
J. G. Downs ${ }^{1}{ }^{1}$ N. D. Smith, ${ }^{1}$ K. K. Mandadapu, ${ }^{2,3}$ J. P. Garrahan, ${ }^{1}$ and M. I. Smith ${ }^{1, *}$ \\ ${ }^{1}$ School of Physics and Astronomy, University of Nottingham, Nottingham, NG7 2RD, United Kingdom \\ ${ }^{2}$ Department of Chemical and Biomolecular Engineering, University of California, Berkeley, California 94720, USA \\ ${ }^{3}$ Chemical Sciences Division, Lawrence Berkeley National Laboratory, Berkeley, California 94720, USA
}

(Received 6 August 2021; accepted 21 October 2021; published 21 December 2021)

\begin{abstract}
We experimentally investigate the nature of 2D phase transitions in a quasi-2D granular fluid. Using a surface decorated with periodically spaced dimples we observe interfacial tension between coexisting granular liquid and crystal phases. Measurements of the orientational and translational order parameters and associated susceptibilities indicate that the surface topography alters the order of the phase transition from a two-step continuous one to a first-order liquid-solid one. The interplay of boundary inelasticity and geometry, either order promoting or inhibiting, controls whether it is the granular crystal or the granular fluid which makes contact with the edge. This order induced wetting has important consequences, determining how coexisting phases separate spatially.
\end{abstract}

DOI: 10.1103/PhysRevLett.127.268002

An understanding of order-disorder phase transitions in two-dimensional systems underpins research in a diverse range of fields, e.g., nanomagnetism, low temperature physics, quantum systems, statistical mechanics, soft matter, plasmas, and molecularly thin films [1-16]. However, many fundamental aspects controlling 2D phase transitions are still not well understood.

In 2D systems, the liquid-solid transition often proceeds via an intermediate hexatic phase, which exhibits quasi-longrange orientational order like a crystal, but short-range positional order like a liquid [17]. Systems with hard discs, are now believed to undergo a continuous crystal-hexatic transition, followed by a first-order hexatic-liquid one $[4,18]$. Yet subtle changes in the interparticle potential can alter this scenario, raising the question of how such theoretical understanding maps onto real world systems [19]. Consequently, recent studies have explored how 2D phase transitions are influenced by factors such as polydispersity, interparticle potential, and shape [19-22]. Experiments have also played a key role, confirming these results and inspiring future research directions [23-25]. A common theme in many of these studies is the idea that preventing 5/7 neighbor disclinations suppresses the hexatic phase leading to a first-order liquid-solid phase transition.

A number of quasi-2D granular studies have observed similarities between their melting behavior and that of hard discs [26-30]. Surprisingly, in these nonequilibrium

Published by the American Physical Society under the terms of the Creative Commons Attribution 4.0 International license. Further distribution of this work must maintain attribution to the author(s) and the published article's title, journal citation, and DOI. systems, the crystal phase generally melts via the twostep continuous Kosterlitz-Thouless-Halperin-Nelson-Young (KTHNY) scenario [31]. However, if the particles are highly inelastic, the liquid-solid phase transition may become first order [29]. Particle inelasticity can also result in wetting by the granular crystal phase. In equilibrium systems, the preferential wetting of a foreign component by the ordered or disordered phase can be governed by geometry rather than interactions, controlling the spatial phase separation at a first-order phase transition $[15,32,33]$. Though this has been demonstrated in simulations of 2D lipid bilayers, such ideas should be generic for order-disorder transitions, both equilibrium and nonequilibrium [32,34].

Two-dimensional systems often interact with an external potential, e.g., a surface, or an electric or magnetic field, which can alter the nature of the observed phase transitions $[6,8,9]$. Early $2 \mathrm{D}$ experiments found that the periodic potential of a commensurate graphite substrate altered the behavior of deposited noble gases $[35,36]$. More recently colloidal experiments have demonstrated that a patterned substrate can control where 2D crystal phases nucleate [37,38]. Simulations of colloids and active matter have also demonstrated how periodic structures can suppress the hexatic and lead to strong phase separation $[39,40]$.

Here we explore how controlling the topography of the surface can be used to manipulate the nature of the underlying liquid-solid transition. We also show how the local structure of the boundaries can be used to spatially control the wetting and phase separation.

Our experiment consists of a partial monolayer of spherical particles $(D=4 \mathrm{~mm})$ on a horizontal metal plate. The plate is subjected to vertical sinusoidal vibrations with a dimensionless acceleration $\Gamma=A(2 \pi f)^{2} / g$ that can be varied in increments of $\Delta \Gamma=0.013$, where $A$ is the 
amplitude and $f$ is the frequency $(50 \mathrm{~Hz})$. As the particles move across the surface in a quasi-2D layer they are filmed from above using a camera (Panasonic HC-X1000, $50 \mathrm{fps}$ ). The location of each particle is measured using the Hough Circle transform (OpenCV) with a precision of $\sim 0.1 \mathrm{~mm}$. In addition to the particle area fraction, $\phi$, the acceleration represents an intensive control variable, playing a role somewhat analogous to the temperature in an equilibrium system. However, the nonequilibrium nature of our experiment introduces important differences. For example, the granular temperature $\left[\sim\left\langle v^{2}\right\rangle\right]$ in coexisting phases is not in general equal as the temperature would be in an equilibrium system [41]. Despite this caveat we will use the language of heating (cooling) to describe increases (decreases) in the acceleration for simplicity.

Two aluminum plates were prepared with different surface topographies: the first flat and the second with a triangular array of dimples (spacing $L \sim 4.8 \mathrm{~mm}$, see Supplemental Material [42]). Our experiment uses custom 3D printed boundaries that are hexagonal in shape (apothem $=100 \mathrm{~mm}$ ). The boundaries are printed with convex dimples on the internal faces with carefully chosen spacings, to which we attach $4 \mathrm{~mm}$ nitrile particles.

We initialize an experiment with $\phi \sim 0.82$ by heating the system to $\Gamma \sim 2.6$. At this acceleration, the entire system exhibits a disordered liquid state. We then slowly cool the system at a rate of $\dot{\Gamma}=0.0013 \mathrm{~s}^{-1}$. At $\Gamma \sim 2.0$ a single crystalline domain surrounded by a disordered phase suddenly forms (see Supplemental Movie 1). Figure 1(a) shows a boundary where the placement of boundary

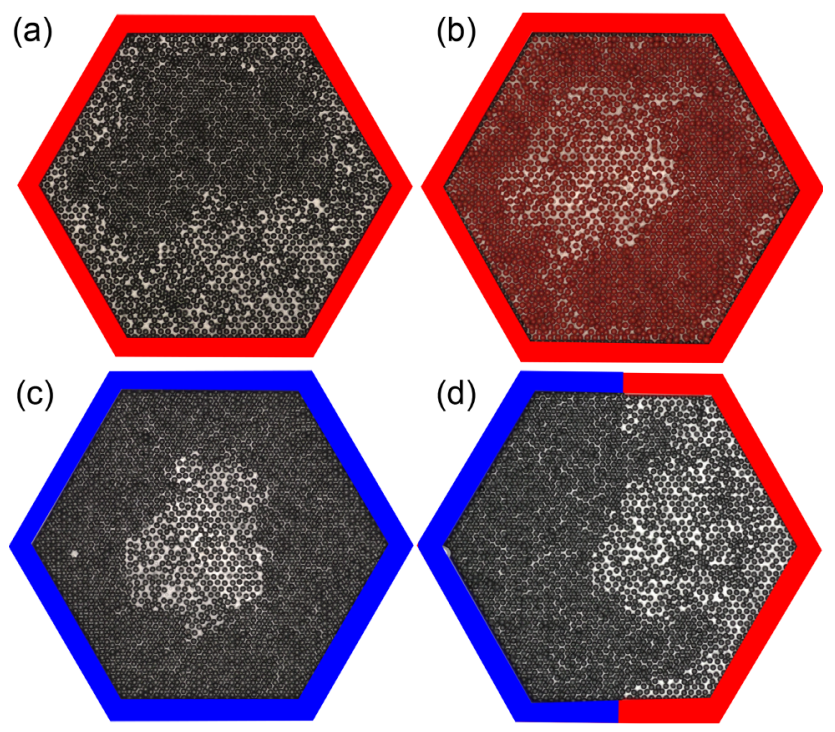

FIG. 1. Wetting of boundary by coexisting phases on a dimpled plate. (a) Inelastic particles with an order-phobic boundary-see definition in text $\left(e_{p p} \sim e_{p w}\right)$. (b) Elastic particles with an orderphobic boundary $\left(e_{p p} \gg e_{p w}\right)$. (c) Inelastic particles with an orderphilic boundary $\left(e_{p p} \sim e_{p w}\right)$. (d) Inelastic particles with a 50:50 orderphobic:orderphillic boundary $\left(e_{p p} \sim e_{p w}\right)$. particles is incommensurate with the observed crystal phase on the dimpled plate ("orderphobic"). Although the region of crystal fluctuates it never wets the boundary, separated by a significant region of liquid phase.

The finite size of some experiments results in the edge playing a significant role in the system's behavior $[43,44]$. Yet even in larger systems the presence of in-plane structures or defects may lead to some spatially varying tendency for one or other phase $[32,39,40,45]$. The black nitrile particles used throughout most of this study are extremely inelastic ( $e \sim 0.1$, see Supplemental Material). In Fig. 1(b) we compare these with more elastic polypropylene particles $(e \sim 0.6)$, though the boundary remains the same. Upon cooling, a crystal phase with the same lattice parameters still forms. One observes competition between frustration of the crystal in the particle layers immediately adjacent to the boundaries and a tendency for the liquid phase to separate to the middle of the experiment. This occurs no matter how slowly the system is cooled, so is not a kinetically trapped configuration but a nonequilibrium steady state.

In a quasi-2D granular experiment, energy enters the system through particles interacting with the vibrating base. At large $\phi$ energy is predominantly transferred and dissipated through interparticle $\left(e_{p p}\right)$ and particle-wall $\left(e_{p w}\right)$ collisions, rather than advection [43]. In Fig. 1(b) $e_{p w} \ll e_{p p}$, therefore the dissipative energy flux near the wall is larger than in the center of the experiment, creating a gradient in the granular temperature [43]. Since the higher density crystal phase has the lower granular temperature, this therefore forms at the edge, only frustrated in the first few layers by the structure of the system boundary. In contrast, in Fig. 1(a) the edge is preferentially wet by the liquid phase. Here, $e_{p w} \sim e_{p p}$ resulting in a more spatially uniform dissipation. This enables the boundary structure to control the location of the different phases. Figure 1(c) confirms this principle, using a boundary structure patterned to be commensurate with the crystalline phase ("orderphillic"). Now the crystal phase wets the boundaries, reversing the spatial phase separation [cf Fig. 1(a)]. This demonstrates that in the absence of strong gradients in dissipation, the wetting can be controlled purely by order or disorder boundary conditions.

Making use of this fact we created a hybrid boundary, where three sides (red) promote disorder and the remaining sides (blue) have been designed to be orderphillic. As we cool the system, the crystalline phase nucleates at the orderphillic boundary and then grows until the contact line becomes pinned at the orderphillic-orderphobic boundary interface [see Fig. 1(d)]. Rotating the ring by $180^{\circ}$, also results in the spatial reversal of the two phases. The liquid and crystal phases are separated by a sharp stable interface $(>72 \mathrm{~h}$ ) which undergoes capillarylike fluctuations, indicating the presence of a significant interfacial tension between the two phases. 

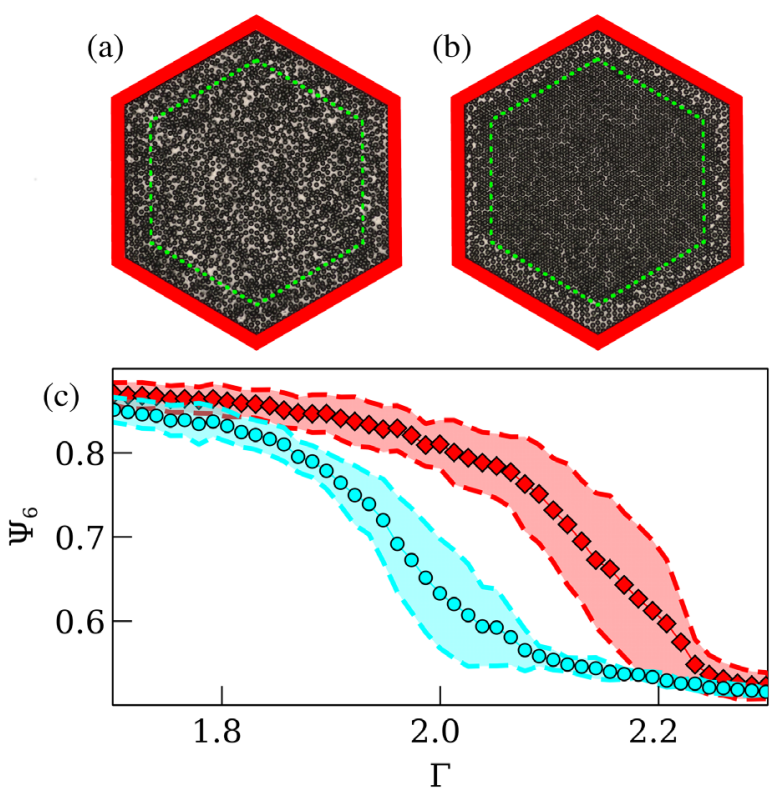

FIG. 2. Hysteresis while heating and cooling using a dimpled plate. Example images (a) $\Gamma \sim 2.4$. (b) $\Gamma \sim 1.7 . \psi_{6}$ is calculated using particles inside the green line. (c) Cooling (blue circles) and heating (red diamonds) at $\dot{\Gamma}=0.0052 \mathrm{~s}^{-1}$.

The presence of a stable interface between two coexisting phases, is strongly indicative of a first-order phase transition [46]. This is surprising as many experimental studies on quasi-2D granulars have found a two-step continuous transition between liquid and crystal [26-30]. However, highly inelastic particles, such as ours, have previously been shown to undergo a first-order phase transition [29]. It is important therefore to establish whether the modified transition is due to the surface dimples or the nature of the particles.

Using purely orderphobic boundaries [Fig. 1(a)] we characterize the system's response to cycles of cooling and heating using both the flat and dimpled surfaces. Starting with a uniform liquid state, $\phi=0.84, \Gamma=2.4$ [Fig. 2(a)] the system is slowly cooled to an acceleration of $\Gamma=1.7$. With the dimpled surface, a single crystalline domain forms, separated from the boundaries due to the wetting properties [Fig. 2(b)]. The system is then slowly heated back to $\Gamma=2.4$. Each experiment is repeated five times.

To characterize the state of the system, we use the global orientational order parameter $\Psi_{6}$. This is the average value of the orientational order parameter $\psi_{6}^{j}$ for each particle $j$ where $\psi_{6}^{j}=\left(1 / n_{j}\right) \sum_{k=1}^{n_{j}} e^{6 i \theta_{j k}} . n_{j}$ is the number of nearest neighbors determined by the Delaunay triangulation and $\theta_{j k}$ is the angle of the vector from particle $j$ to neighboring particle $k . \Psi_{6}$ is calculated using those particles away from the edge [green line in Figs. 2(a) and 2(b)]. Figure 2(c) shows, for the dimpled plate, how the value of $\Psi_{6}(\Gamma)$ changes as the system is cooled (blue circles) and heated
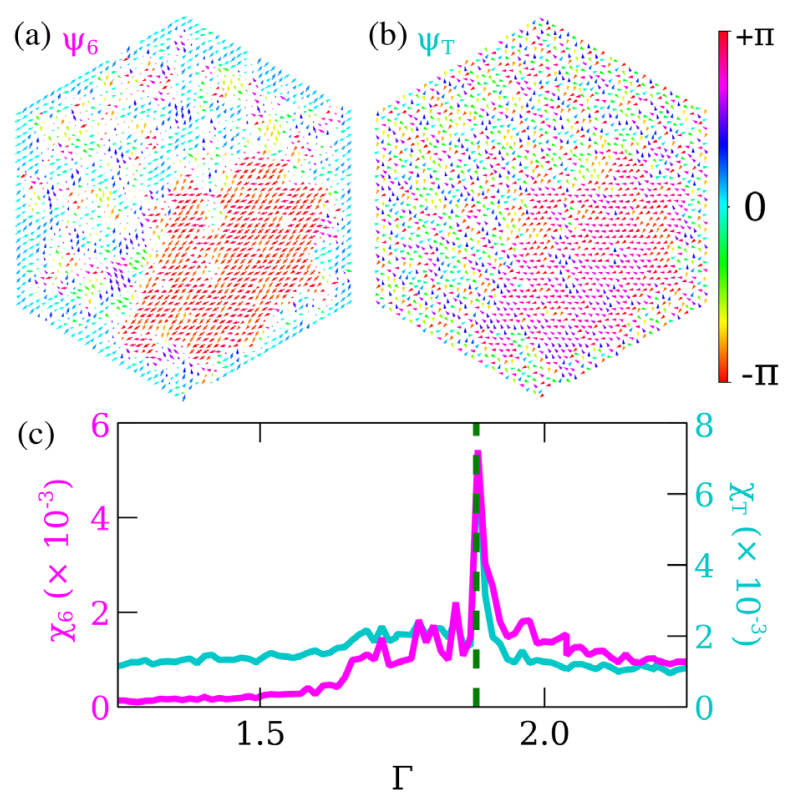

FIG. 3. First-order phase transition with decreasing $\Gamma$ on a dimpled plate. Upper panels show a vector representation of the complex order parameters (a) orientational $-\psi_{6}$ and (b) translational $-\psi_{T}$ at $\Gamma=1.9$, cooling at $\dot{\Gamma} \sim 2.2 \times 10^{-4} \mathrm{~s}^{-1}$ (see main text for definitions). The presence of a single ordered domain separated from a liquid phase is clearly visible. (c) The susceptibility $\chi$ characterizes the size of fluctuations in each order parameter. The maximum in $\chi_{6}$ (magenta) and $\chi_{T}$ (cyan) occur at the same value of $\Gamma$ indicating a first order phase transition.

(red diamonds) at a rate of $\dot{\Gamma}=5.2 \times 10^{-3} \mathrm{~s}^{-1}$. The standard deviation of repeat experiments is indicated by the shaded areas surrounding each curve.

When using the dimpled plate a pronounced hysteresis is observed [Fig. 2(c)] which depends on the cooling rate (Supplemental Fig. 3). The presence of hysteresis suggests the order-disorder transition is first order [47]. To confirm that the hysteresis is not due to the high particle inelasticity of our particles [29] but is caused by the dimpled surface, we repeated the experiment using the flat plate. Supplemental Fig. 4 shows that despite using inelastic particles, experiments using the flat plate exhibited no hysteresis. This indicates that the first-order characteristics arise due to the dimpled surface topography.

In liquids, orientational and translational order are short range, while in crystals they are both quasilong range. In 2D, KTHNY theory predicts two separate transitions separated by an intermediate hexatic phase characterized by quasilong range orientational order, but short-range translational order [31]. In contrast, in a one-step first-order phase transition the growth of both types of order occurs together.

The local translational order of a $2 \mathrm{D}$ system is characterized by the order parameter $\psi_{T}^{j}=e^{i \vec{G} \cdot \vec{r}_{j}}$, where $\vec{r}_{j}$ is the position vector of particle $j$ and $\vec{G}$ is a primary reciprocal lattice vector calculated from the Delaunay tessellation. Calculating $\psi_{6}^{j}$ and $\psi_{T}^{j}$ for each particle, we plot the 

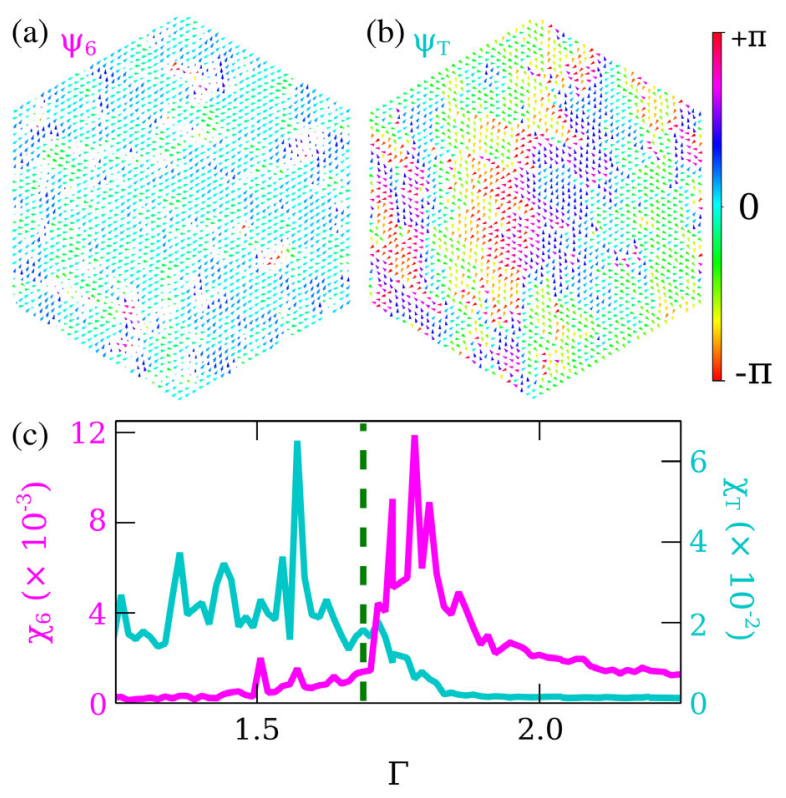

FIG. 4. Two-step phase transition with decreasing $\Gamma$ on a flat plate. Upper panels show a vector representation of the complex order parameters (a) orientational $-\psi_{6}$ and (b) translational $-\psi_{T}$ at $\Gamma=1.7$, cooling at $\dot{\Gamma} \sim 2.2 \times 10^{-4} \mathrm{~s}^{-1}$ (see main text for definitions). No correlations are observed between the two vector fields. (c) The susceptibility $\chi$ characterizes the size of fluctuations in each order parameter. The maximums in $\chi_{6}$ (magenta) and $\chi_{T}$ (cyan) occur at different values of $\Gamma$ indicating a two-step transition with an intervening hexatic phase.

corresponding vector fields, Figs. 3(a) and 3(b), respectively. Longer arrows in the orientational order vector reflect a more ordered configuration, with the angle and color related to the orientation of the measured hexagonal order. The length of the translational order vector is always 1 , while the angle and color represent the degree of translational order, such that $\pm \pi$ corresponds to particles positioned at the expected lattice points of the crystal. Plotting the vector fields allows us to visually compare changes in the spatial correlations of each order parameter for both the dimpled and flat plate (Figs. 3 and 4, Supplemental Movies 2 and 3).

Measurements of the spatial decay of correlations in the order parameters are often used in simulations to assess where transitions occur $[5,9,11]$. However, in experiments, finite size and time effects can introduce ambiguities. Co-existing crystal and liquid phases can also result in a power-law decay that mimics a hexatic phase [34]. To avoid these difficulties, we measured the susceptibility of these order parameters [19, 34]. The orientational (translational) susceptibilities are defined as $\chi_{6(T)}=$ $\left\langle\left|\Psi_{6(T)}^{2}\right|\right\rangle-\left\langle\left|\Psi_{6(T)}\right|\right\rangle^{2}$, where $\Psi_{6(T)}$ is the average order parameter of the system in each case. The susceptibility characterizes the size of the fluctuations in an order parameter and exhibits a maximum at a phase transition.

For the dimpled plate, as the system is slowly cooled, both orientational and translational susceptibilities exhibit a single maximum at the same value of the acceleration, $\Gamma \sim 1.9$ [Fig. 3(c)]. At this critical acceleration a large area of the orientational and translational vector fields suddenly becomes spatially correlated, indicating the formation of a crystalline nucleus [Figs. 3(a) and 3(b)]. This nucleus remains stable with fluctuations both spatially and temporally synchronized (see Supplemental Movie 2). In addition to our earlier results, this is compelling evidence that the dimpled surface results in a first-order phase transition from liquid-solid without an intervening hexatic phase.

Performing the same experiment using the flat plate, the maximum in the fluctuations of each order parameter occur separately, indicating two separate transitions for the orientational $\Gamma \sim 1.78$ and the translational susceptibility $\Gamma \sim 1.57$ [Fig. 4(c)]. As the system cools through these transitions one observes a gradual increase in the length scale of spatial fluctuations in the orientational order parameter [Fig. 4(a)]. The spatial length scale of the translational order parameter also gradually grows as the system is cooled. However, unlike the dimpled plate, the changes of both order parameters are continuous and there are no clear spatial correlations between the two (see Supplemental Movie 3).

The presence of two distinct peaks suggests our experiment on the flat plate undergoes a two-step transition consistent with other quasi-2D granular studies [27-30]. In addition, we were unable to find evidence of a finite surface tension in this system. This suggests that in our experiments on the flat plate, contrary to recent equilibrium results on colloids [24], the liquid-hexatic transition is a continuous two step transition. Importantly, in the context of this work it confirms that the first-order phase transition observed with the dimples does not merely strengthen a first-order liquid-solid phase transition, ultimately arising from particle inelasticity, but fundamentally alters the nature of the transition.

In our experiments using a flat plate, highly inelastic particles $(e \sim 0.1)$ resulted in a two-step phase transition. This is different from the results of Komatsu et al. [29] who found that the inelasticity of their particles $(e \sim 0.1)$ resulted in a first-order phase transition. Subtle differences in granular experiments can radically alter observed phase behavior [48]. Indeed, the Komatsus experiment differs from ours in having a confining lid. However, this raises the question of whether inelasticity alone is sufficient to change the order of the transition, something which warrants further investigation.

The introduction of a dimpled surface breaks both the orientational and translational symmetry of the $2 \mathrm{D}$ particle fluid. However, this is only significant if the kinetic energy at which the particles undergo a liquid-solid transition is sufficiently small to be influenced by the underlying topography. The situation is like equilibrium scenarios involving noble gases on an underlying graphite lattice. There the hexatic intermediate is not observed if the liquidsolid transition occurs at thermal energies comparable to 
the substrate potential [49]. If the dimples are significant, both orientational and translational ordering are affected together resulting in a single transition. One might also think that the creation of a pentagonal/heptagonal disclination, essential to the KTHNY scenario, would be much more difficult in the presence of a surface that encourages hexagonal ordering.

In this Letter we have considered the importance of orderphilic and orderphobic controlled wetting. We demonstrated that a dimpled surface can result in a first-order order-disorder transition. However, there is an important additional consequence when these phenomena occur together. In Fig. 1(d) the use of a hybrid boundary that is orderphillic and orderphobic resulted in the symmetry of the system being broken. The order induced wetting therefore controlled the location of the resultant coexisting phases. As a system phase separates during a first-order order-disorder phase transition, order induced wetting can therefore have a long-range effect, controlling the final spatial composition of a system. Our work therefore demonstrates experimentally the importance of topography in controlling order induced interactions in $2 \mathrm{D}$ phase transitions.

We thank S. Devlin and J. Pearson for technical support with equipment development. J.G. D. acknowledges a $\mathrm{PhD}$ studentship supported by the Royal Society. N. D. S. acknowledges a PhD studentship supported by the Engineering and Physical Sciences Research Council [Grant No. EP/M506588/1]. J. P. G. acknowledges support from the EPSRC [Grant No. EP/R04421X/1]. M. I. S. gratefully acknowledges a Royal Society University Research Fellowship.

*mike.i.smith@nottingham.ac.uk

[1] W. Li, X. Qian, and J. Li, Nat. Rev. Mater. 6, 829 (2021).

[2] X. Wang, Z. Song, W. Wen, H. Liu, J. Wu, C. Dang, M. Hossain, M. A. Iqbal, and L. Xie, Adv. Mater. 31, 1804682 (2019).

[3] J.-M. Zanotti, P. Judeinstein, S. Dalla-Bernardina, G. Creff, J.-B. Brubach, P. Roy, M. Bonetti, J. Ollivier, D. Sakellariou, and M.-C. Bellissent-Funel, Sci. Rep. 6, 25938 (2016).

[4] E. P. Bernard and W. Krauth, Phys. Rev. Lett. 107, 155704 (2011).

[5] M. Durand and J. Heu, Phys. Rev. Lett. 123, 188001 (2019).

[6] J. Rysti, J. Mkinen, S. Autti, T. Kamppinen, G. Volovik, and V. Eltsov, Phys. Rev. Lett. 127, 115702 (2021).

[7] V. Nosenko, S. K. Zhdanov, A. V. Ivlev, C. A. Knapek, and G. E. Morfill, Phys. Rev. Lett. 103, 015001 (2009).

[8] D. Miserev, J. Klinovaja, and D. Loss, Phys. Rev. B 103, 024401 (2021).

[9] P. Matvija, F. Rozbořil, P. Sobotík, I. Oštádal, B. Pieczyrak, L. Jurczyszyn, and P. Kocán, Sci. Rep. 7, 7357 (2017).
[10] I. Guillamón, H. Suderow, A. Fernández-Pacheco, J. Sesé, R. Córdoba, J. M. D. Teresa, M. R. Ibarra, and S. Vieira, Nat. Phys. 5, 651 (2009).

[11] S. Haravifard, A. Banerjee, J. C. Lang, G. Srajer, D. M. Silevitch, B. D. Gaulin, H. A. Dabkowska, and T.F. Rosenbaum, Proc. Natl. Acad. Sci. U.S.A. 109, 2286 (2012).

[12] P. Simon and D. Loss, Phys. Rev. Lett. 98, 156401 (2007).

[13] A. R. Kolovsky, Phys. Rev. A 93, 033626 (2016).

[14] R. L. C. Vink, Phys. Rev. Lett. 98, 217801 (2007).

[15] J. G. Dash, H. Fu, and J. S. Wettlaufer, Rep. Prog. Phys. 58, 115 (1995).

[16] W. Freyl and, Phys. Chem. Chem. Phys. 10, 923 (2008).

[17] B. Halperin and D. R. Nelson, Phys. Rev. Lett. 41, 121 (1978).

[18] M. Engel, J. A. Anderson, S. C. Glotzer, M. Isobe, E. P. Bernard, and W. Krauth, Phys. Rev. E 87, 042134 (2013).

[19] S. C. Kapfer and W. Krauth, Phys. Rev. Lett. 114, 035702 (2015).

[20] J. Russo and N. B. Wilding, Phys. Rev. Lett. 119, 115702 (2017).

[21] J. A. Anderson, J. Antonaglia, J. A. Millan, M. Engel, and S. C. Glotzer, Phys. Rev. X 7, 021001 (2017).

[22] Y.-W. Li and M. P. Ciamarra, Phys. Rev. Lett. 124, 218002 (2020).

[23] G. Briand and O. Dauchot, Phys. Rev. Lett. 117, 098004 (2016).

[24] A. L. Thorneywork, J. L. Abbott, D. G. A. L. Aarts, and R. P. A. Dullens, Phys. Rev. Lett. 118, 158001 (2017).

[25] B. Li, X. Xiao, S. Wang, W. Wen, and Z. Wang, Phys. Rev. X 9, 031032 (2019).

[26] J. S. Olafsen and J. S. Urbach, Phys. Rev. Lett. 81, 4369 (1998).

[27] J. S. Olafsen and J. S. Urbach, Phys. Rev. Lett. 95, 098002 (2005).

[28] P. M. Reis, R. A. Ingale, and M. D. Shattuck, Phys. Rev. Lett. 96, 258001 (2006).

[29] Y. Komatsu and H. Tanaka, Phys. Rev. X 5, 031025 (2015).

[30] X. Sun, Y. Li, Y. Ma, and Z. Zhang, Sci. Rep. 6, 24056 (2016).

[31] D. R. Nelson, Defects and Geometry in Condensed Matter Physics (Cambridge University Press, Cambridge, England, 2002).

[32] S. Katira, K. K. Mandadapu, S. Vaikuntanathan, B. Smit, and D. Chandler, eLife 5, 13150 (2016).

[33] A. Archer and A. Malijevsky, J. Phys. Condens. Matter 28, 244017 (2016).

[34] S. Katira, J. P. Garrahan, and K. K. Mandadapu, Phys. Rev. Lett. 120, 260602 (2018).

[35] R. J. Birgeneau, G. S. Brown, P. M. Horn, and D. E. Moncton, J. Phys. C 14, L49 (1981).

[36] K. J. Strandburg, Rev. Mod. Phys. 60, 161 (1988).

[37] R. Ganapathy, M. R. Buckley, S. J. Gerbode, and I. Cohen, Science 327, 445 (2010).

[38] C. K. Mishra, A. K. Sood, and R. Ganapathy, Proc. Natl. Acad. Sci. U.S.A. 113, 12094 (2016).

[39] W. Qi and M. Dijkstra, Soft Matter 11, 2852 (2015). 
[40] C. Reichhardt and C. J. O. Reichhardt, Phys. Rev. E 103, 022602 (2021).

[41] J. P. D. Clewett, R. M. Bowley, and M. R. Swift, Phys. Rev. Lett. 123, 118001 (2019).

[42] See Supplemental Material at http://link.aps.org/ supplemental/10.1103/PhysRevLett.127.268002 for annotated movie of crystal formation and animated plots of the orientational (translational) order parameters. Additional details concerning the creation of dimpled surfaces. Supplemental plots.

[43] N. D. Smith and M. I. Smith, Phys. Rev. E 96, 062910 (2017).
[44] F. Pacheco-Vázquez, G. A. Caballero-Robledo, and J. C. Ruiz-Suárez, Phys. Rev. Lett. 102, 170601 (2009).

[45] P. Galajda, J. Keymer, P. Chaikin, and R. Austin, J. Bacteriol. 189, 8704 (2007).

[46] L.-H. Luu, G. Castillo, N. Mujica, and R. Soto, Phys. Rev. E 87, 040202(R) (2013).

[47] J. V. Selinger, Introduction to the Theory of Soft Matter (Springer International Publishing, New York, 2016).

[48] G. Castillo, N. Mujica, and R. Soto, Phys. Rev. Lett. 109, 095701 (2012).

[49] G. Aeppli and R. Bruinsma, Phys. Rev. Lett. 53, 2133 (1984). 